\title{
Aph-1 Is Required to Regulate Presenilin-Mediated $\gamma$-Secretase Activity and Cell Survival in Drosophila Wing Development
}

\author{
Emilie Cooper ${ }^{1,2}$, Wu-Min Deng ${ }^{3}$, and Hui-Min Chung ${ }^{1,{ }^{*}}$ \\ ${ }^{1}$ Department of Biology, University of West Florida, Pensacola, Florida \\ ${ }^{2}$ Environmental Protection Agency, Gulf Ecology Division, Gulf Breeze, Florida \\ ${ }^{3}$ Department of Biological Science, Florida State University, Tallahassee, Florida
}

\section{Summary}

\begin{abstract}
Aph-1 is a multipass transmembrane protein and an essential component of the Presenilin (Psn)mediated $\gamma$-secretase complex. During protease assembly, Aph-1 stabilizes the newly synthesized Psn holoprotein to facilitate generation of the active form of Psn, which is a Psn-NTF/Psn-CTF heterodimer produced through a Presenilinase-initiated endoproteolytic cleavage of the Psn holoprotein. Although it is clear that loss of Aph-1 activity leads to failure of Psn heterodimer formation, little is understood about whether Aph-1 plays a role in regulating $\gamma$-secretase activity in addition to assisting Psn maturation. Using various modified Psn forms that do not require endoproteolysis or have a large deletion of the cytosolic loop, we show that in Drosophila Aph-1 is still required for $\gamma$-secretase activity independent of its role in promoting Psn endoproteolysis. In addition, our results indicate that Aph-1 is required to promote cell survival in the wing imaginal disc; aph-1 mutant cells are lost either through cell death or because of a defect in cell proliferation. This function of Aph-1 is independent of its role in regulating $\gamma$-secretase activity, but possibly involves downregulating the activity of uncleaved Psn holoprotein.
\end{abstract}

\section{Keywords}

Presenilin; Aph-1; $\gamma$-secretase; Notch; cell survival

\begin{abstract}
$\gamma$-Secretase is an evolutionarily conserved protease that cleaves type I integral membrane proteins including the amyloid precursor protein (APP) and Notch proteins (Dries and $\mathrm{Yu}$, 2008; Iwatsubo, 2004). It is a high molecular weight complex that contains at least four basic components: Presenilin (Psn), Nicastrin (Nct), Aph-1, and Pen-2 (Dries and Yu, 2008; Iwatsubo, 2004). One key event during protease assembly is Presenilinase-mediated endoproteolysis of the newly synthesized Psn holoprotein to form the mature Psn-NTF/PsnCTF heterodimer-the catalytic subunit of the protease. Biochemical studies have shown that Aph-1 and Nct form a subcomplex to stabilize the Psn holoprotein prior to Psn endoproteolysis that is regulated by Pen-2 (Dries and Yu, 2008; Iwatsubo, 2004). Loss of Aph-1 functions leads to failure of production of active Psn and subsequently $\gamma$-secretase activity, but it is not clear whether Aph-1 plays a role in regulating $\gamma$-secretase activity post-Psn maturation (Francis et al., 2002; Goutte et al., 2002; Hu and Fortini, 2003; Iwatsubo, 2004).
\end{abstract}


In an accompanying study (Barakat et al., 2009), we show that Psn endoproteolysis is not essential for forming a functional Psn protein in Drosophila by using two approaches. Firstly, by simultaneously expressing two constitutively active transgenes, $P s n^{N T F}$ and $P s n^{C T F}$, which encode Psn-NTF and Psn-CTF respectively, we showed that the reconstituted Psn ${ }^{\mathrm{NTF}}$ / Psn ${ }^{\mathrm{CTF}}$ activity was able to substitute for endogenous Psn activity. Secondly, we showed that $\mathrm{Psn}^{\Delta \mathrm{exon} 9}$, which is an endoproteolysis-deficient Psn protein, could also substitute for endogenous Psn activity. To examine whether, in addition to assisting the Psn endoproteolysis event, Aph-1 plays a role in mediating $\gamma$-secretase activity, we examined the requirement of Aph-1 in the presence of either the reconstituted Psn ${ }^{\mathrm{NTF}} / \mathrm{Psn}^{\mathrm{CTF}}$ or $\mathrm{Psn}^{\Delta \mathrm{exon} 9}$ proteins during fly development. Since Psn-mediated $\gamma$-secretase activity is required for proper processing of the Notch receptor, animals with loss of $\gamma$-secretase would show phenotypes similar to those of loss of Notch signaling (Struhl and Greenwald, 1999). We used assays that monitor events of Notch signaling to examine the phenotype of aph-1 ${ }^{-1-}$ cells in the presence of either the reconstituted $\mathrm{Psn}^{\mathrm{NTF}} / \mathrm{Psn}^{\mathrm{CTF}}$ or $\mathrm{Psn}^{\Delta \mathrm{exon} 9}$ proteins.

Previously, Hu and Fortini showed that flies lacking Aph-1 function exhibited phenotypes similar to those of loss of Notch signaling, aph-1 ${ }^{-l-}$ embryos develop extensive neural hyperplasia, and clones of aph-1 $1^{-/-}$cells gave rise to a notched wing phenotype in adult flies (Hu and Fortini, 2003). Using mosaic analysis, we generated clones of $a p h-1^{-/-}$cells in flies that express the reconstituted $\mathrm{Psn}^{\mathrm{NTF}} / \mathrm{Psn}{ }^{\mathrm{CTF}}$ or $\mathrm{Psn}^{\Delta \mathrm{exon} 9}$ in addition to endogenous Psn. In the presence of reconstituted $\mathrm{Psn}^{\mathrm{NTF}} / \mathrm{Psn}{ }^{\mathrm{CTF}}$ activity, clones of $a p h-1^{-/-}$cells in the wing exhibited notched wing and thickened wing vein phenotypes typically associated with loss of Notch signaling (Fig. 1d). Similar wing phenotypes were also found associated with aph-1 mutant cells in wings constitutively expressing $\operatorname{Psn}^{\Delta \operatorname{exon} 9}$ (Fig. 1f). These results are consistent with the idea that, post-Psn endoproteolysis, Aph-1 activity is still needed for Psn-mediated $\gamma$-secretase cleavage of Notch protein. To confirm this, we then examined the ability of $a p h-1^{-/-}$cells in the wing disc to transduce Notch signals by characterizing expression of known Notch target genes. This was again done in the absence and the presence of either the reconstituted $\mathrm{Psn}^{\mathrm{NTF}} / \mathrm{Psn}^{\mathrm{CTF}}$ or $\mathrm{Psn}^{\Delta \mathrm{exon} 9}$ activities.

During wing development, Notch signaling is required for $\mathrm{D} / \mathrm{V}$ boundary formation in the wing disc by regulating gene expression of $c u t(c t)$ and wingless $(w g)$; loss of Notch signaling at the D/V boundary can be traced by loss of $c t$ or $w g$ expression (Blair, 1996; Micchelli et al., 1997; Rulifson et al., 1996; Schweisguth, 2004). In this study, aph-1+- cells were marked with $1 \times$ GFP expression, clones of $a p h-1^{-l-}$ cells with the absence of GFP expression, and their adjacent twin spot clones of $a p h-1^{+/+}$cells with $2 \times$ GFP expression. Since $a p h-I^{-/-}$and $a p h-1^{+/+}$cells were derived simultaneously from a cell division following an FLP-FRTinduced mitotic recombination, each twin spot of $a p h-1^{+/+}$should be accompanied by an aph- $-^{-/-}$mutant clone (Xu and Rubin, 1993).

When examining mosaic wing discs, we were surprised to observe that clones of $a p h-1^{-1-}$ cells were actually difficult to locate. Frequently, clones of $a p h-1^{-1-}$ cells were missing from their adjacent twin spots of $a p h-I^{+/+}$cells. Most of the wing discs analyzed had several twin spots but no $a p h-1^{-/-}$mutant clones (Fig. 2a). In fact, of 21 twin spots identified, only two had mutant clones associated (Table 1), suggesting most of $a p h-1^{-/-}$cells do not survive in the wing epithelium. The exact reason for this remains to be determined. Aph-1 could be required to maintain normal proliferation; a reduction would result in loss of the clones by competition. Alternatively, Aph-1 could be required to maintain viability of the cells, which are lost simply because they die (it is unlikely that the clones are lost by extrusion of the mutant cells from the epithelium, as we have not identified cells in the lumen of the disc, although this is still a possibility if the cells die after extrusion). Not knowing the exact cause of the cell growth and/ or proliferation problem of $a p h-I^{-/}$cells, we hereby refer the problem loosely as a survival problem. We then performed a mosaic analysis of $a p h-1^{-1-}$ cells in the presence of Psn ${ }^{+}-\mathrm{HA}$, 
which can substitute for endogenous Psn but as expected did not rescue phenotypes caused by loss of Aph-1 function: the majority of twin spots did not have mutant clones associated (only six mutant clones compared to 40 twin spots, Table 1). Overall, less than $20 \%$ of $a p h-1^{+/+}$twin spots were associated with $a p h-1^{-/-}$mutant clones in the wing discs of flies expressing wild type Psn.

We next compared $\mathrm{aph}-\mathrm{I}^{-{ }_{-1}}$ mutant clones to those of $\mathrm{Psn}^{-{ }_{-}^{-}}$and $n c t^{-1-}$ to determine if the survival defect in aph-1 ${ }^{-/-}$cells observed in wing disc can be explained simply by loss of $\gamma$ secretase activity. We found that, in contrast to those of $a p h-1^{-/-}$cells, the majority of $\mathrm{Psn}^{-l-}$ and $\mathrm{nct}^{-{ }^{--}}$cells did survive and were able to proliferate. Of $29 \mathrm{Psn}^{+/+}$twin spots, 20 had $\mathrm{Psn}^{-/-}$mutant clones associated, and of $26 \mathrm{nct}^{+/+}$twin spots, 20 had $\mathrm{nct}^{-/-}$mutant clones associated. Clones of $n c t^{-1-}$ cells, marked by the lack of GFP expression, were frequently detected adjacent to their twin spots of $n c t^{+/+}$cells that were marked by expression of $2 \times$ GFP (Fig. 2b), so were $P_{s n^{-1-}}$ cells (Fig. 2c). These results imply that Aph-1 plays a role in positively regulating cell survival in the wing disc through a mechanism independent of its role in forming an early subcomplex with Nct during assembly of the $\gamma$-secretase complex.

It is conceivable that the role of Aph-1 in regulating cell survival is independent of its role in assisting Psn endoproteolysis and subsequent $\gamma$-secretase activity. We tested this by looking at whether aph-1-1- mutant cells survive normally in the presence of the modified forms of Psn - the form that bypasses Psn endoproteolysis (reconstituted $\mathrm{Psn}^{\mathrm{NTF}} / \mathrm{Psn}^{\mathrm{CTF}}$ ) and the form that cannot be cleaved by Presenilinase $\left(\operatorname{Psn}^{\Delta \operatorname{exon} 9}\right)$. We then compared the phenotype of aph $-1^{-1-}$ cells that express Psn ${ }^{\Delta \text { exon9 }}$ to those that express reconstituted Psn ${ }^{\mathrm{NTF}} / \mathrm{Psn}{ }^{\mathrm{CTF}}$, both in the context that all cells still express endogenous Psn. We found that, like $a p h-1^{-/-}$cells, $a p h-1^{-/-}$cells constitutively expressing Psn ${ }^{\Delta \text { exon9 }}$ also had the defect in cell survival. Only about $21 \%$ of twin spots were associated with $a p h-1^{-1-}$ mutant clones (Table 1). In contrast, when provided with the reconstituted $\mathrm{Psn}^{\mathrm{NTF}} / \mathrm{Psn}^{\mathrm{CTF}}$ activity, the majority of twin spots had aph-1 $1^{-/-}$clones associated (about $84 \%$ shown in Table 1). However, although aph-1 $1^{-/-}$mutant cells appeared to survive and proliferate almost normally in the presence of reconstituted the $\mathrm{Psn}^{\mathrm{NTF}} / \mathrm{Psn}{ }^{\mathrm{CTF}}$ activity, they did not have the ability to transduce Notch signaling, as judged by loss of $c t$ expression at the D/V boundary (Fig. 3b).

Thus, although neither $\mathrm{Psn}^{\Delta \text { exon9 }}$ nor the reconstituted $\mathrm{Psn}^{\mathrm{NTF}} / \mathrm{Psn}{ }^{\mathrm{CTF}}$ could rescue loss of Notch signaling phenotypes caused by the absence of Aph-1 activity, they differentially affect the ability of aph-1 $\mathrm{I}^{-/-}$cells to survive in the wing disc. Since both $\mathrm{Psn}^{\Delta \mathrm{exon} 9}$ and reconstituted $\mathrm{Psn}^{\mathrm{NTF}} / \mathrm{Psn}{ }^{\mathrm{CTF}}$ are mature Psn forms but differ in that $\mathrm{Psn}^{\Delta \mathrm{exon} 9}$ has an unbroken cytosolic loop and the reconstituted $\mathrm{Psn}^{\mathrm{NTF}} / \mathrm{Psn}^{\mathrm{CTF}}$ does not, it is conceivable that it is the presence of an intact loop in Psn that is influencing cell survival in the absence of Aph-1 activity. We tested this by analyzing whether $a p h-1^{-1-}$ mutant cells can survive in the presence of an active Psn form that has a large deletion of the cytosolic loop, $\mathrm{Psn}^{\Delta l o o p}$.

In Barakat et al. (2009), we showed that Psn ${ }^{\Delta l o o p}$, a modified Psn form with a deletion of a large part of the cytosolic loop between TMD6 and TMD7, could substitute for the endogenous Psn activity. We were curious to learn whether $a p h-1^{-/-}$cells that had Psn ${ }^{\Delta l o o p}$ activity could transduce Notch signaling and whether they would survive. Again, using mosaic analysis, we generated clones of $a p h-1^{-1-}$ cells in flies that express Psn ${ }^{\Delta l o o p}$ in addition to endogenous Psn. We found that, in the presence of the Psn ${ }^{\Delta l o o p}$ activity, clusters of $a p h-1^{-{ }^{-}}$cells in the wing still exhibited notched wing and thickened wing vein phenotypes typically associated with loss of Notch signaling (Fig. 1h). Approximately $92 \%$ of expected clones of $a p h-1^{-1-}$ cells were detected adjacent to their twin spots in the wing disc (see Table 1) in the presence of the $\mathrm{Psn}^{\Delta l o o p}$ activity, but mutant clones that crossed the D/V boundary lacked $c t$ expression (Fig. 3c). Thus, although Psn ${ }^{\Delta l o o p}$ activity cannot rescue loss of Notch signaling in $a p h-1^{-/-}$cells, like reconstituted $\mathrm{Psn}{ }^{\mathrm{NTF}} / \mathrm{Psn}{ }^{\mathrm{CTF}}$, it does promote their survival in the disc. 
Overall, our results show that, $a p h-1^{-/-}$cells, which still express the endogenous Psn ${ }^{+}$ holoprotein, often fail to survive in the wing disc (Fig. 2a and Table 1), but an artificial addition of either reconstituted $\mathrm{Psn}^{\mathrm{NTF}} / \mathrm{Psn}^{\mathrm{CTF}}$ or $\mathrm{Psn}^{\Delta \text { loop }}$ (but not $\mathrm{Psn}^{\Delta \text { exon9 }}$ ) can overcome the survival defect of $a p h-1^{-1-}$ cells (Fig. 3 and Table 1). These observations lead us to propose that, the $\mathrm{Psn}^{+}$holoprotein, which still possesses an intact cytosolic loop has an activity that either antagonizes cell proliferation or possibly promotes cell death. In aph-1 mutant cells, this form will accumulate and this may account for the loss of aph-1 mutant clones. In the future, we would like to test this hypothesis by examining the survival of aph-1 ${ }^{-/-} \mathrm{Psn}^{-/-}$cells, the prediction being that double mutant clones should behave like $P s n^{-/-}$and not $a p h-1^{-/-}$clones because any $\mathrm{Psn}^{+}$holoprotein will now be absent. In addition, we should point out that our hypothesis has to further propose that Psn forms that do not have an intact cytosolic loop can override the antagonistic activity of the $\mathrm{Psn}^{+}$holoprotein, although potential mechanisms will await further analysis of this phenomenon. The evidence for this is that the survival defect of $a p h 1^{-/-}$cells that still express endogenous Psn is not rescued by Psn ${ }^{\Delta e x o n 9}$, which retains the cytosolic loop, but is rescued by $\mathrm{Psn}^{\mathrm{NTF}} / \mathrm{Psn}^{\mathrm{CTF}}$ and $\mathrm{Psn}^{\Delta l o o p}$, in which the loop is either cleaved or absent.

We should also point out that $a p h-1^{--}$cells do survive and proliferate in some situations. In the wing, a small percentage of clones do survive and proliferate even in the presence of only wild-type Psn; the reduction in the number of clones indicates that this ability is reduced compared to wild-type cells. Also, Hu and Fortini showed aph- $1^{-1-}$ embryos that have the maternal Aph-1 contribution removed developed extensive neural hyperplasia, indicating aph- $1^{-/-}$cells still proliferate during embryonic development (Hu and Fortini, 2003). It is unclear if their proliferative ability is reduced in embryos as it is in imaginal wing discs.

In summary, using various modified Psn forms, we show that Aph-1 regulates $\gamma$-secretase activity independent of any role it may have in promoting Psn endoproteolysis. One possibility is that it remains an integral part of $\gamma$-secretase after Psn endoproteolysis and is required for the activity of the complex (Dries and Yu, 2008). Alternatively, it might be involved in other processes such as trafficking of $\gamma$-secretase within the cell, potentially even prior to Psn proteolysis. Its exact role will require further analysis. Further, our observations indicate that Psn holoprotein, unregulated by Aph-1, may antagonize cell survival, at least in some tissues. Psn-mediated $\gamma$-secretase activity is well conserved among animal species. How the $\gamma$-secretase activity is regulated has been a significant question for developmental biologists and for researchers working on human diseases, especially those related to neurodegeneration. Previous studies show that, in addition to its interactions with Aph-1, Nct, and Pen-2, Psn also interacts with a number of other proteins (Chen and Schubert, 2002; Parks and Curtis, 2007). However, little is understood whether these interactions affect Psn functioning relevant to $\gamma$ secretase activity, and with what form of Psn that these interactions take place. The various Psn forms used here would be instrumental to investigate the complex behaviors of Psn.

\section{METHODS}

\section{Transgenes}

All the Psn transgenes used here were discussed in detail in Barakat et al. (2009). Psn transgenes encoding the various Drosophila Psn forms were expressed under the control of the Tubulin $\alpha 1$ (tub) promoter. Psn forms encoded by the transgenes of $t u b-P s n^{+}$-HA (Chung and Struhl, $2001), t u b-P s n^{\Delta \text { exon9 }}$, and $t u b-P s n^{\mathrm{CTF}}$ are tagged with two copies of the HA epitope inserted between the Gly361 and Gln362 residues. Psn form encoded by the $t u b-P s n^{\mathrm{NTF}}$ transgene has three copies of myc epitope tag inserted between the Ser46 and Ser47 residues. Psn form encoded by the $t u b-P s n^{\Delta l o o p}$ transgene has the amino acids $326-433$ of the predicted Psn large cytosolic loop being replaced with six copies of myc epitope tag. Psn form encoded by the $t u b-P s n^{\Delta \text { exon9 }}$ transgene has the amino acids 313-342 of Psn removed. 


\title{
Fly Stocks and Mosaic Analysis
}

The aph-1 $1^{D 35}$ FRT40, Psn ${ }^{c 2}$ ri FRT2A, and FRT82B e $n c t^{R 46}$ chromosomes were used to investigate phenotypes of flies lacking Aph-1, Psn, and Nct functions, respectively (Chung and Struhl, 2001; Hu and Fortini, 2003; Struhl and Greenwald, 1999, and personal communication). For the sake of simplicity, cells that are homozygous $P s n^{c 2}, a p h-1^{\mathrm{D} 35}$, and $n c t^{R 46}$ were

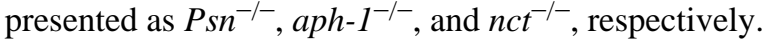

Clones of homozygous mutant cells were generated by hs-flp/FRT-induced mitotic recombination in the second or early third instar of larvae with a heat shock at $35^{\circ} \mathrm{C}$ for 60 $\mathrm{min}$, and were analyzed in imaginal discs or adult flies (Xu and Rubin, 1993). Fly strains of y w hs-flp; hs-GFP y+ FRT40/CyO, y w hs-flp; ubi-GFP FRT40/CyO, y w hs-flp; ubi-GFP FRT2A/TM6b, and $y w h s$-flp; FRT82 ubi-GFP/TM6b were used to provide female parents of mosaic analysis.

All the analysis of aph-1 mutant clones was done in the homozygous $P s n^{+/+}$or heterozygous $\mathrm{Psn}^{-/+}$background. Genotypes of fly strains that were used for mosaic analysis of $a p h-I^{-/-}$ cells are listed below:
1. y w hs-flp; aph-1 ${ }^{\mathrm{D} 35}$ FRT40/CyO; MKRS/TM6b
2. y w hs-flp; aph-1 ${ }^{\mathrm{D} 35}$ FRT40/CyO; $\mathrm{Psn}^{\mathrm{c} 2}$ ri FRT2A/TM6b
3. y w hs-flp; aph-1 ${ }^{\mathrm{D} 35}$ FRT40/CyO; $\mathrm{Psn}^{\mathrm{c} 2}$ ri FRT2A tub-Psn ${ }^{+}-\mathrm{HA} / \mathrm{TM} 6 \mathrm{~b}$
4. y w hs-flp; aph-1 ${ }^{\mathrm{D} 35}$ FRT40/CyO; $\mathrm{Psn}^{\mathrm{c} 2}$ ri (FRT2A) tub-Psn ${ }^{\Delta e x o n 9} / \mathrm{TM} 6 \mathrm{~b}$
5. y w hs-flp; aph-1 ${ }^{\mathrm{D} 35}$ FRT40/CyO; $\mathrm{Psn}^{\mathrm{c} 2}$ ri (FRT2A) tub-Psn ${ }^{\Delta l o o p} / \mathrm{TM} 6 \mathrm{~b}$
6. $\mathrm{y}$ w hs-flp; tub - Psn ${ }^{\mathrm{NTF}}$ aph-1 ${ }^{\mathrm{D} 35}$ FRT40/CyO; $\mathrm{Psn}^{\mathrm{c} 2}$ ri (FRT2A) tub-Psn ${ }^{\mathrm{CTF}} / \mathrm{TM} 6 \mathrm{~b}$

\section{Analysis of Adult Wings and Immunostaining}

Wings from adult flies were mounted in GMM. Imaginal discs were fixed and stained by standard protocols using mouse anti-Ct (MAb 2B10 supernatant, 1:50, Developmental Studies Hybridoma Bank) (Struhl and Greenwald, 2001). Images were analyzed using a Leica confocal microscope or a Leica DMLB microscope associated with the SPOTBASIC software.

\section{ACKNOWLEDGMENTS}

\begin{abstract}
We thank Mark Fortini, Bloomington Stock Center, and Developmental Studies Hybridoma Bank for providing fly stocks and antisera. We thank current and past members in Chung's lab for their help in fly work. We are grateful to Deborah Chapman, Gerard Campbell, and anonymous reviewers for their comments on this manuscript. Cooper was a graduate student of the master program at the Department of Biology in the University of West Florida.

Contract grant sponsor: NIH (AREA R15 grant), Contract grant number: 1R15AG028448-01 (to H-MC), Contract grant sponsor: University of West Florida.
\end{abstract}

\section{LITERATURE CITED}

Barakat A, Mercer B, Cooper E, Chung H-M. Examing requirement for formation of functional presenilin proteins and their processing events in vivo. Genes J Genet Dev. 2009doi: 10.1002/DVG20475

Blair SS. Notch and wingless signals collide. Science 1996;271:1822-1823. [PubMed: 8596948]

Chen Q, Schubert D. Presenilin-interacting proteins. Expert Rev Mol Med 2002;4:1-18. [PubMed: 14585160]

Chung HM, Struhl G. Nicastrin is required for Presenilin-mediated transmembrane cleavage in Drosophila. Nat Cell Biol 2001;3:1129-1132. [PubMed: 11781576]

Dries DR, Yu G. Assembly, maturation, and trafficking of the gamma-secretase complex in Alzheimer's disease. Curr Alzheimer Res 2008;5:132-146. [PubMed: 18393798] 
Francis R, McGrath G, Zhang J, Ruddy DA, Sym M, Apfeld J, Nicoll M, Maxwell M, Hai B, Ellis MC, Parks AL, Xu W, Li J, Gurney M, Myers RL, Himes CS, Hiebsch R, Ruble C, Nye JS, Curtis D. aph-1 and pen-2 are required for Notch pathway signaling, gamma-secretase cleavage of betaAPP, and presenilin protein accumulation. Dev Cell 2002;3:85-97. [PubMed: 12110170]

Goutte C, Tsunozaki M, Hale VA, Priess JR. APH-1 is a multipass membrane protein essential for the Notch signaling pathway in Caenorhabditis elegans embryos. Proc Natl Acad Sci USA 2002;99:775779. [PubMed: 11792846]

$\mathrm{Hu}$ Y, Fortini ME. Different cofactor activities in gamma-secretase assembly: evidence for a nicastrinAph-1 subcomplex. J Cell Biol 2003;161:685-690. [PubMed: 12771124]

Iwatsubo T. The gamma-secretase complex: Machinery for intra-membrane proteolysis. Curr Opin Neurobiol 2004;14:379-383. [PubMed: 15194119]

Micchelli CA, Rulifson EJ, Blair SS. The function and regulation of cut expression on the wing margin of Drosophila: Notch, Wingless and a dominant negative role for Delta and Serrate. Development 1997;124:1485-1495. [PubMed: 9108365]

Parks AL, Curtis D. Presenilin diversifies its portfolio. Trends Genet 2007;23:140-150. [PubMed: 17280736]

Rulifson EJ, Micchelli CA, Axelrod JD, Perrimon N, Blair SS. Wingless refines its own expression domain on the Drosophila wing margin. Nature 1996;384:72-74. [PubMed: 8900280]

Schweisguth F. Notch signaling activity. Curr Biol 2004;14:R129-R138. [PubMed: 14986688]

Struhl G, Greenwald I. Presenilin is required for activity and nuclear access of Notch in Drosophila. Nature 1999;398:522-525. [PubMed: 10206646]

Struhl G, Greenwald I. Presenilin-mediated transmembrane cleavage is required for Notch signal transduction in Drosophila. Proc Natl Acad Sci USA 2001;98:229-234. [PubMed: 11134525]

Xu T, Rubin GM. Analysis of genetic mosaics in developing and adult Drosophila tissues. Development 1993;117:1223-1237. [PubMed: 8404527] 

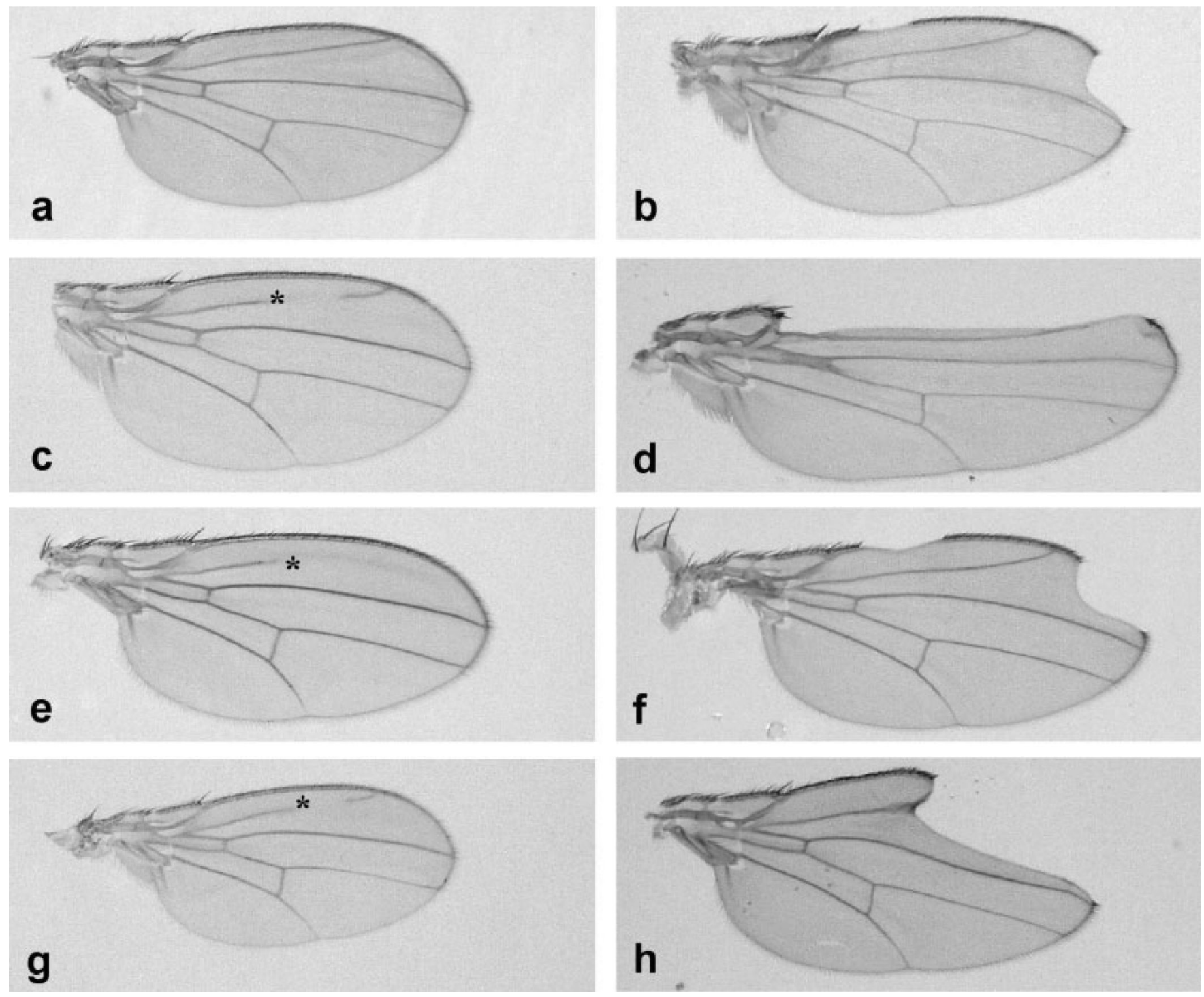

FIG. 1.

Aph-1 is still needed to mediate Notch signaling in flies that express reconstituted $\mathrm{Psn}^{\mathrm{NTF}}$ / Psn ${ }^{\mathrm{CTF}}, \mathrm{Psn}^{\Delta \mathrm{exon} 9}$, or Psn ${ }^{\Delta l o o p}$. Wings of wild type fly (a), Psn mutant flies rescued by expression of reconstituted $P s n^{N T F} / P s n^{C T F}(\mathbf{c})$, of $P s n^{\Delta e x o n 9}(\mathbf{e})$, or of $P s n^{\Delta l o o p}$ (g). Mosaic analysis of $a p h-1^{-1-}$ cells in the heterozygous $P s n^{-/+}$flies that express no $P s n$ transgene (b), reconstituted $P s n^{N T F} / P s n^{C T F}(\mathbf{d}), P s n^{\Delta e x o n 9}(\mathbf{f})$, or $P s n^{\Delta l o o p}(\mathbf{h})$. (The asterisk signs in c, e, and $\mathrm{g}$ indicate broken wing vein II phenotype due to recessive homozygous ri. All the cells in c, e, and $g$ are homozygous $r i$ and homozygous $P s n^{c 2}$.) 

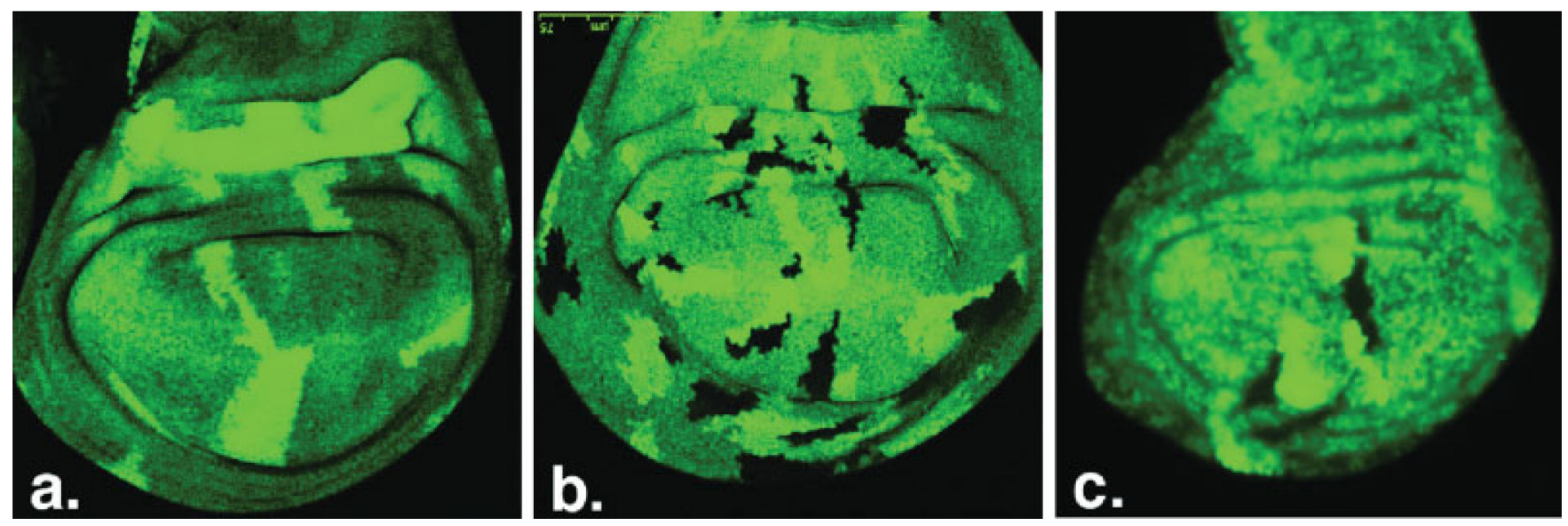

FIG. 2.

Behavior of $a p h-1^{-1-}, n c t^{-1-}$, and $P s n^{-1-}$ clones in the wing disc. Mosaic analysis of aph $-1^{-/-}$(a), $n c t^{-/-}$cells (b), and $\mathrm{Psn}^{-/-}$cells (c) in the $a p h-1^{-/+}, n c t^{-/+}$, and $P s n^{-/+}$flies, respectively, in which the homozygous mutant cells are marked by the absence of GFP, the twin clones composed of homozygous wild type cells by the expression of $2 \times$ GFP, and their surrounding heterozygous mutant cells by the expression of $1 \times$ GFP. 

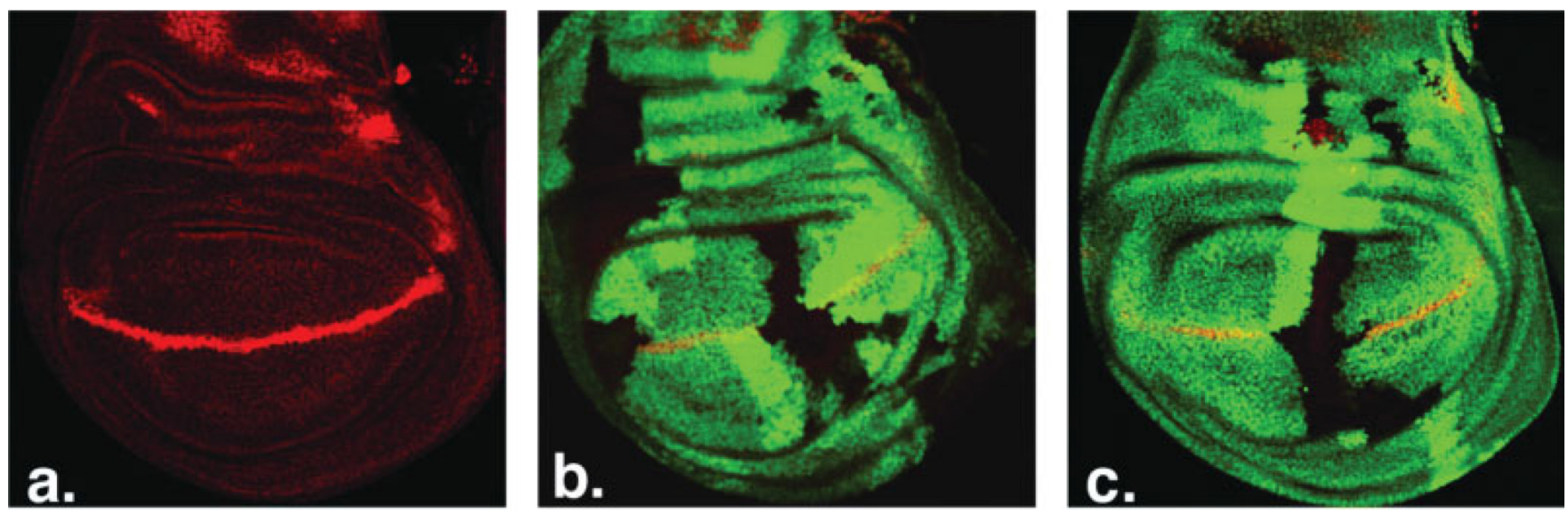

FIG. 3.

Aph $-1^{-I-}$ cells proliferate in the presence of reconstituted $\mathrm{Psn}^{\mathrm{NTF}} / \mathrm{Psn}^{\mathrm{CTF}}$ or $\mathrm{Psn}^{\Delta \mathrm{loop}}$ in the wing disc. Mosaic analysis of aph-1 $I^{-/-}$clones in the heterozygous $\mathrm{Psn}^{-/+}$flies that express reconstituted $\mathrm{Psn}^{\mathrm{NTF}} / \mathrm{Psn}^{\mathrm{CTF}}(\mathbf{b})$ or $\mathrm{Psn}^{\Delta l o o p}(\mathbf{c})$. The $a p h-1^{-l-}$ cells are marked by the absence of GFP, the twin clones composed of homozygous wild type cells by the expression of $2 \times$ GFP, and their surrounding heterozygous mutant cells by the expression of $1 \times$ GFP. Expression of ct (red) in the D/V boundary of the wing disc is absent in aph-1 $1^{-/-}$cells in (b). and (c); the expression of $\mathrm{ct}$ in the $a p h-1^{-/+}$wing disc is referenced (a). 


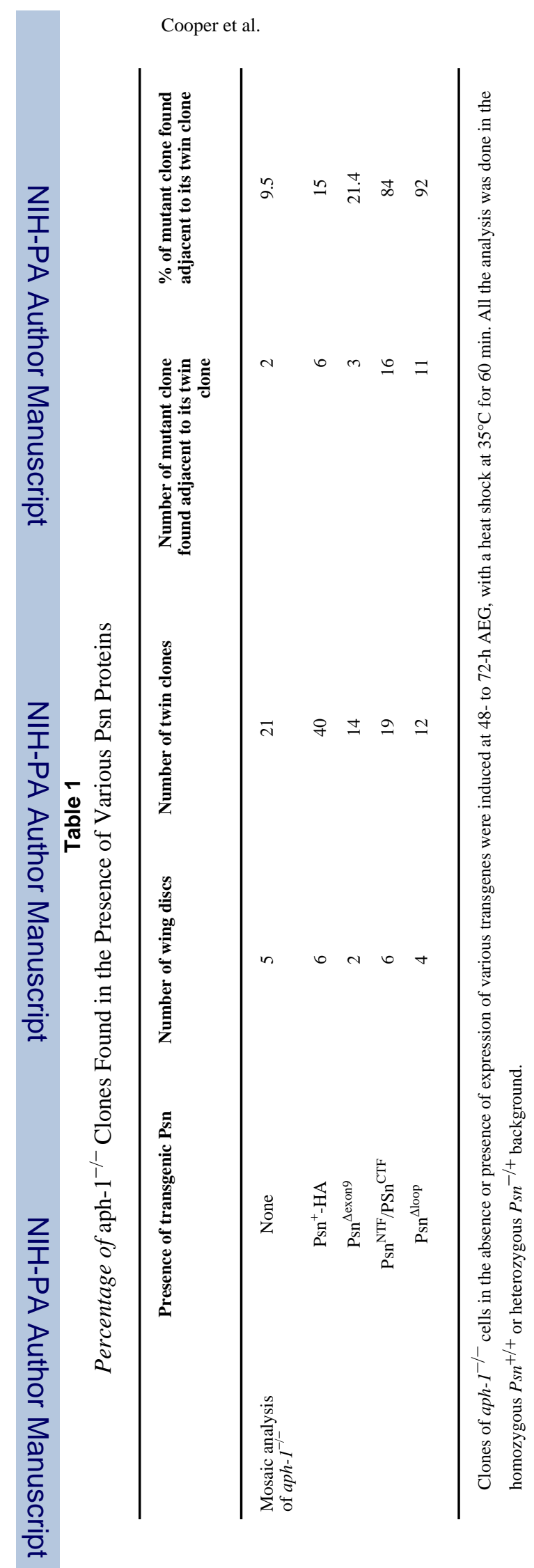

Genesis. Author manuscript; available in PMC 2009 September 24. 